\title{
Improving After-Clinic Work Workflow in a Family Medicine Residency
}

\author{
Nguyen $V^{*}$, J aqua E, Oh A, Altamirano $M$ and \\ J aqua $\mathbf{T}$ \\ Department of Family Medicine, Loma Linda University \\ Health, USA \\ *Corresponding author: Van Nguyen, Department of \\ Family Medicine, Loma Linda University Health, 1200 \\ California Street, Suite 240, Redlands, CA 92374, USA
}

Received: May 08, 2021; Accepted: June 03, 2021; Published: June 10, 2021

\begin{abstract}
Introduction: The broad range of patients and diagnoses addressed by primary care physicians lends to a larger after-work clinic load. The resulting after-clinic work, including various in-basket tasks, can be a substantial burden to physicians, and potentially leading to burnout. The goal of this study is to generate a standardized workflow to improve physician after-clinic work efficiency and patient care.
\end{abstract}

Methods: A nine-question pre- and post-intervention survey about afterclinic work management was administered to family medicine residents at a multi-specialty FQHC in California. The intervention was done in June 2020 and included a twenty-minute training session explaining how to implement a standardized in-basket management flowchart in a family medicine residency clinic.

Results: Pre- and post-intervention data were analyzed using nonindependent paired sample t-tests. The survey was sent to all 40 family medicine residents. Pre- and post-intervention survey response rate was $77.5 \%$ and $97.5 \%$ respectively.

The result of the nine questions post intervention were statistically significant ( $p$ value of $<0.001$ ). The standardized flowchart addressed adequate supervision of resident physicians' patient care.

Conclusion: The post-intervention results showed that having a clear and standardized flowchart enhanced the overall knowledge and understanding by the resident physicians in how to management the in-basket workflow. With increased patient access via telehealth and enhanced electronic medical records, it is essential to have effective teaching and supervision of resident physician after-clinic work. Successful teaching of after-clinic work will improve work-life balance and the overall success of the new primary care physician.

\section{Introduction}

Although in-basket work is noted in all specialties, the volume is significantly larger for Primary Care Physicians (PCP) who treat broader patient populations and diseases. PCPs have been estimated to spend 66.8 minutes per day on in-basket management [1]. This is a significant burden that is added to each work day and, although perceived as administrative duty, after-clinic work, such as in-basket management, is essential to timely patient care and a physicians' well-being [2]. Boohaker et al have noted that proper follow-up and documentation is not always consistent and can increase the risk for suboptimal patient care and malpractice litigation [3]. With such an important role in patient care, it is interesting that many clinics do not have detailed workflows or specific steps for in-basket management [4].

Residency clinics such as the family medicine residency at a Federally Qualified Health Center (FQHC) in San Bernardino, California highlights this need for detailed in-basket management. This clinic serves a medically underserved and economically diverse community that requires care both in and out of the clinic [5]. The clinic is situated in a community that is predominantly Hispanic/ Latino with one-fifth of the population who do not have a high school education. Specifically, the community is $53.4 \%$ Hispanic/Latino, $42 \%$ non-English speaking, $20.8 \%$ do not complete high school, and $18 \%$ live below the poverty line [6,7]. Patients often lack the means necessary for proper communication, such as stable housing, and may have transportation barriers in coming to clinic.

Residency often focuses on developing medically and procedurally competent physicians but fails to recognize that the residents have a unique experience in the outpatient clinic as they navigate after-clinic work. A resident physician's schedule is often unpredictable, continuity with patients is not ideal, and a certain level of supervision is needed at all times. This can lead to inadequate preparation to manage the after-clinic work, potentially lead to delays in patient care, and bad outcomes from overlooked critical results. Thus, creating a workflow for in-basket management was needed in order for resident physicians to continue their patients' competent and proficient care while having the appropriate supervision from their attending physicians.

Physician in-basket management is a critical skill that should be honed through residency training as a resident physician progressively gains independence in direct patient care and management. Teaching in-basket management will ensure that resident physicians will 
Table 1: Pre- and Post- Intervention Survey.

On a scale of 1 to 5 , with 1 indicating "strongly disagree" and 5 indicating "strongly agree", please rate the following statements.

\begin{tabular}{|l|l|}
\hline \multicolumn{2}{|c|}{ On a scale of $\mathbf{1}$ to $\mathbf{5}$, with $\mathbf{1}$ indicating "strongly disagree" and $\mathbf{5}$ indicating "strongly agree", please rate the following statements. } \\
\hline Statement 1 & I know how to address non-urgent abnormal in-basket results. \\
\hline Statement 2 & I know how to address (know who to notify) urgent abnormal in-basket results. \\
\hline Statement 3 & I know how to address (know who to notify) urgent abnormal in-basket results after hours. \\
\hline Statement 4 & I feel comfortable addressing patient advice request through MyChart. \\
\hline Statement 5 & I know when it is appropriate to address patients' advice requests through MyChart. \\
\hline Statement 6 & I know when it is expected of me to route a result or request to an Attending. \\
\hline Statement 7 & I know how to address medication refill requests for controlled substances. \\
\hline Statement 8 & I know how to address general medication refill requests. \\
\hline Statement 9 & I know how to address a medication refill request for a patient who has not been seen in over 3 months. \\
\hline
\end{tabular}

Table 2: Two-Tailed Paired Samples t-Test for the Difference Between Pre-Test and Post-Test.

\begin{tabular}{|c|c|c|c|c|c|c|}
\hline \multicolumn{2}{|c|}{ Pre-Test } & \multicolumn{2}{c|}{ Post-Test } & & & \\
\hline M & SD & M & SD & t & p & d \\
\hline 2.28 & 0.1 & 3.8 & 0.27 & -15.73 & $<0.001$ & 4.97 \\
\hline
\end{tabular}

Note: $\mathrm{N}=10$. Degrees of Freedom for the t-statistic $=9 . \mathrm{d}$ represents Cohen's $\mathrm{d}$

provide timely and proper patient management while mitigating the potential for burnout from never-ending after-clinic work.

This study implemented a standardized workflow to optimize efficient and patient-centered management of various in-basket tasks, while allowing for supervision of resident physicians in a family medicine residency clinic. This study aimed to clarify each member of the healthcare team's role in after-clinic work and provide consistent feedback to resident physicians in their patient care. This ensured resident physician education and patient safety. The intangible aims included furthering resident physician education, improving worklife balance, increasing satisfaction at work, and decreasing physician burnout

\section{Methods}

Utilizing a Likert scale, a nine-question pre- and post-intervention survey was distributed to family medicine residents at a large, urban, multi-specialty FQHC in San Bernardino, California, exploring multiple aspects of after-clinic work including management of test results, patient messages, and medication refills (Table 1 and Table 2). A standardized in-basket management flowchart was created and distributed to resident physicians and teaching faculty over a twentyminute training session (Figures 1-3). Teaching faculty assisted in the implementation of the flowchart in the residency clinic and residents were surveyed in one month regarding its usability and relevance to training. This study was done in June 2020 with the goal of establishing a workflow prior to the new academic year.

\section{Results}

Pre- and post-intervention data were analyzed using two-tailed paired sample t-tests. The survey was sent by email trough the Loma Linda University Health Qualtrics survey engine to all 40 family medicine residents. Pre-intervention survey response rate was $77.5 \%$

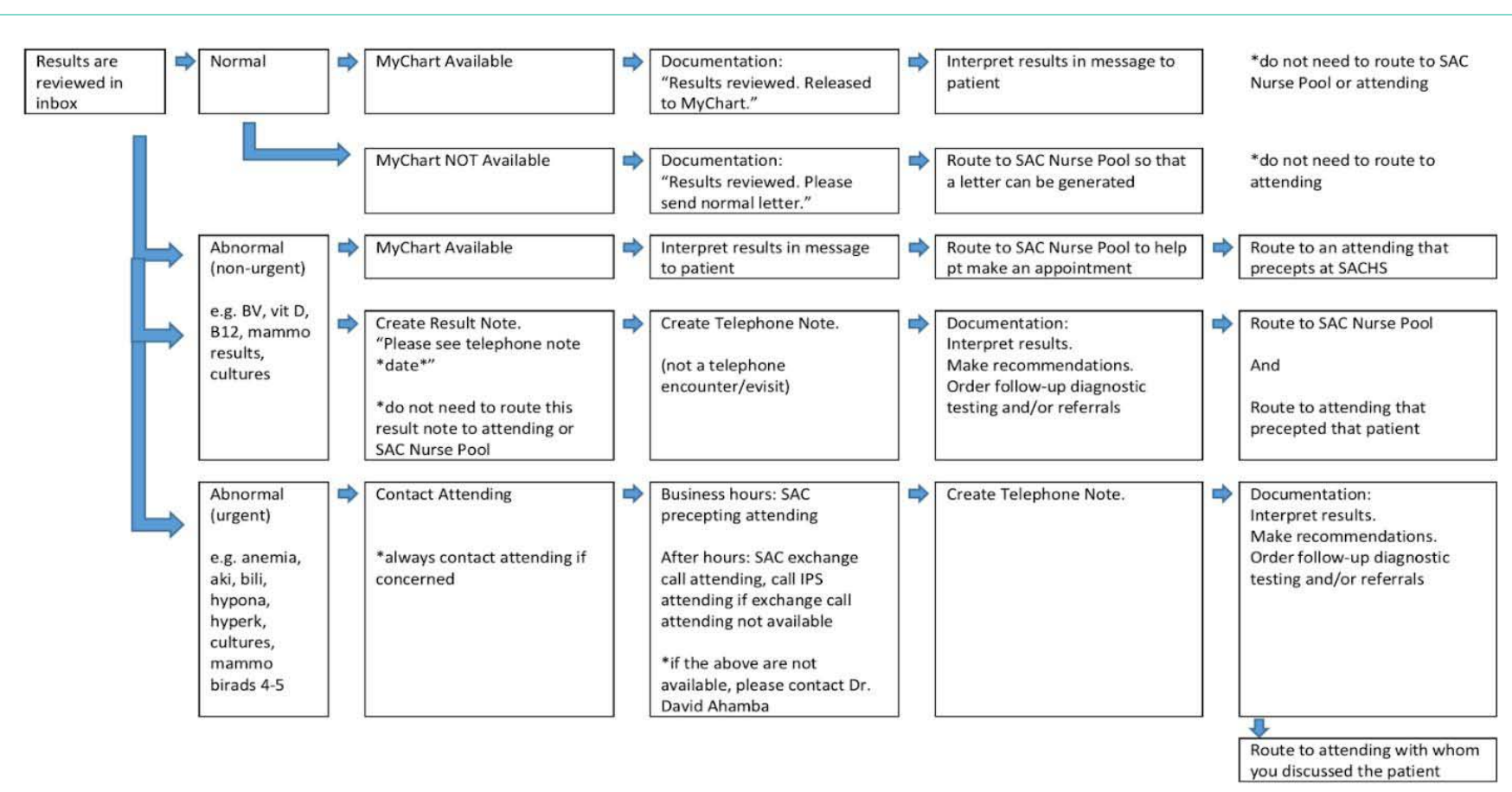

Figure 1: Flowchart for the management of test results. 


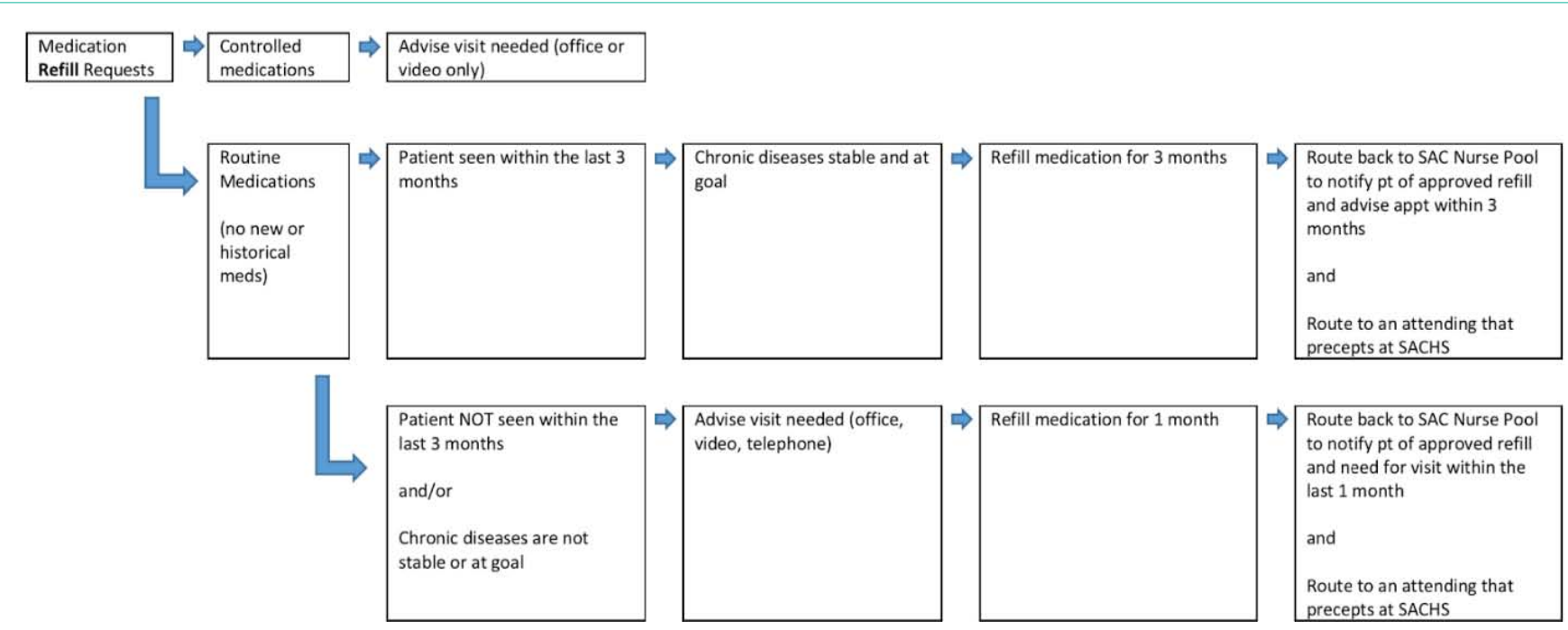

Figure 2: Flowchart for the management of medication refill requests.

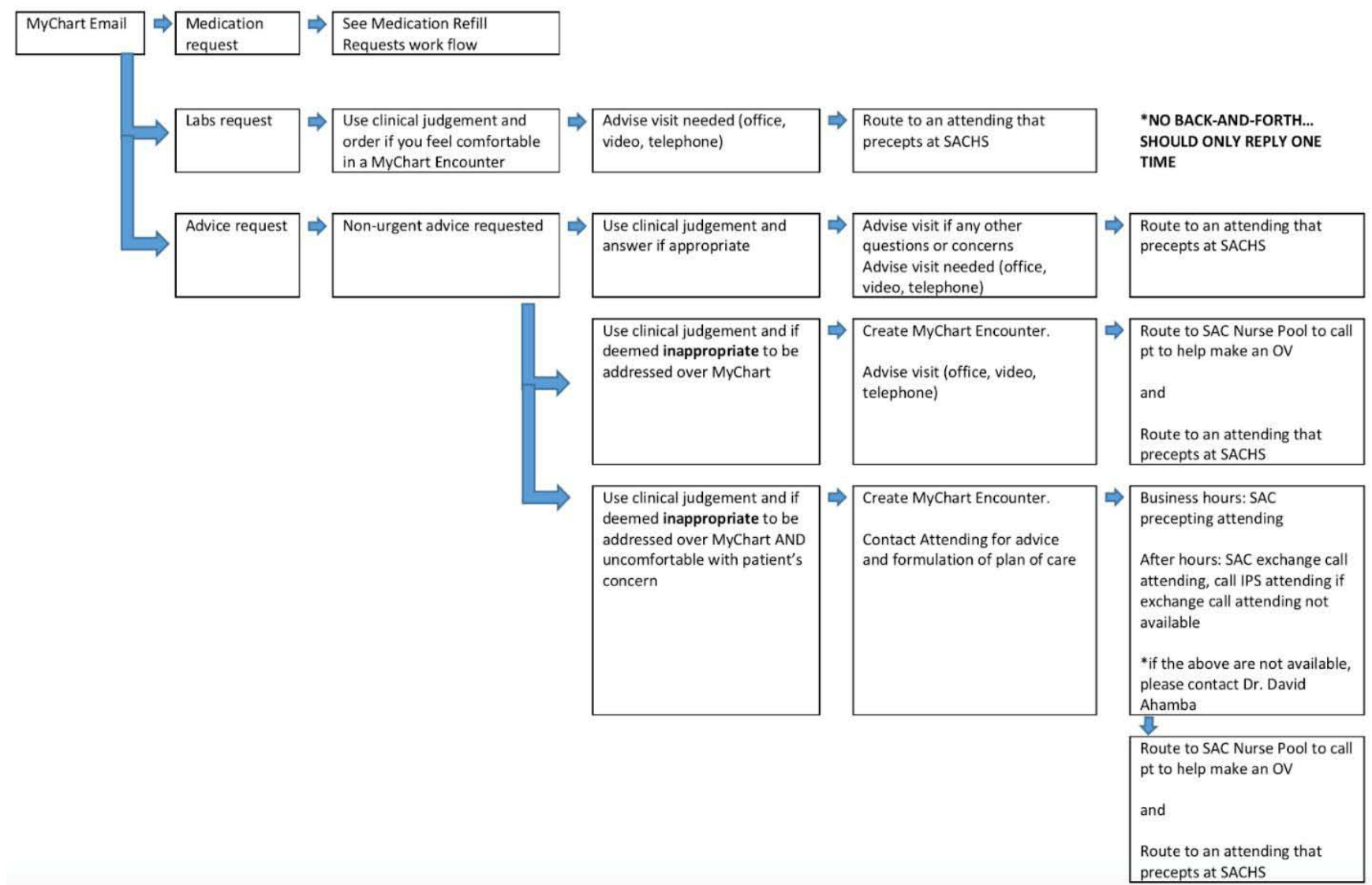

Figure 3: Flowchart for the management of patient messages.

and $97.5 \%$ for the post-intervention survey. These paired sample $\mathrm{t}$-tests, with a $\mathrm{p}$ value of $<0.001$, for each question were statistically significant. This demonstrates that addressing common in-basket tasks in a clear flowchart improved knowledge and management of said tasks. See Table 2 for a summary of all results.

\section{Discussion}

\section{Overview}

Acknowledging that clinicians spend over one hour a day managing in-basket tasks, the need to be efficient and proficient in completing these is essential to becoming a successful and well- 
rounded primary care physician [1]. The purpose of this study is to address the unique need of residency clinics in completing after-clinic work by implementing a standardized flowchart in a family medicine residency clinic.

\section{Study limitations}

Although the data suggests that the structured flowchart increases resident physicians' competency and supervision of the management of common in-basket tasks, the study is limited by the small sample size and short duration of the study. The study was in a medium-sized family medicine residency clinic, housed in an FQHC. Thus, it is not clear if the results of this study can be extrapolated to significantly larger or smaller residency programs or non-primary care specialtybased clinics.

\section{Multi-faceted approach}

Although providing education and adequate supervision to resident physicians regarding the management of in-basket tasks was a primary goal of this study, the ultimate goal was to provide competent and efficient care to patients. A test is only useful if the results are reviewed and translated into action, not just lost in the electronic medical record [8]. It has already been noted that the lack of closed-loop communication with patients can lead to increased litigation, so, engaging the patient in their own care was of the utmost importance [3]. The most important aspect of the in-basket flowchart was to establish clear recommendations of when a patient should make an appointment to discuss their concerns. The flowchart may have emphasized the resident physicians' role in in-basket tasks, but these tasks are insurmountable without faculty, scheduling, messaging unit, nursing, and patient engagement.
With more enhanced electronic medical records, asynchronous care is becoming a more pronounced form of patient care, one that is unlikely temporary. Although it has improved access for patients, it has led to more after-clinic work [1]. This burden necessitates both proficient and efficient in-basket management-a burden that should be addressed as early as possible in a physician's training. Equipping physicians, as early as possible in their training, with the tools needed to complete these important tasks are essential to developing successful and well-rounded primary care physicians. A clear flowchart, as implemented in this study, can help resident physicians appropriately address in-basket tasks, ensure proper supervision for patient care, and help reduce some of the stress and burnout associated with after-work care.

\section{References}

1. Murphy D, et al. The Burden of Inbox Notifications in Commercial Electronic Health Records. JAMA Intern Med. 2016; 176: 559-560.

2. Tai-Seale M, et al. Physicians' Well-Being Linked to In-Basket Messages Generated By Algorithms in Electronic Health Rewards. Health Affairs. 2019; 38: 1073-1078.

3. Boohaker EA, et al. Patient notification and follow-up of abnormal test results. A physician survey. Arch Intern Med. 1996; 156: 327-331.

4. Elder N, et al. Management of Test Results in Family Medicine Offices. Ann of Fam Med. 2009; 7: 343-351.

5. U.S. Health and Human Services, Health Resources and Administration Services. 2021

6. San Bernardino QuickFacts from the U.S. Census Bureau 2013-2017. 2021.

7. DATA USA: San Bernardino County, CA. 2021.

8. Bodley $\mathrm{T}$, et al. Test Result Management Practices of Canadian Internal Medicine Physicians and Trainees. J Gen Intern Med. 2019; 34: 118-124. 